\title{
Allergenauswahl schrumpft
}

- Viele Therapieallergene müssen nach der Therapieallergene-Verordnung vom 13. November 2008 zukünftig Zulassungsprüfungen durchlaufen. Betroffene Allergene sind alle Süßgräser außer Mais, Frühblüher (Birke, Erle, Hasel), Dermatophagoides sp. sowie Bienen- und Wespengift. Die Allergenhersteller mussten dem Paul-EhrlichInstitut (PEI) bis Mai 2009 anzeigen, ob sie eine Zulassung für solche Allergene und Allergenmischungen anstreben. Alle Allergene, für die keine Zulassung angestrebt wird, sind nur noch bis November 2011 verordnungsfähig, berichtete Prof. Ludger Klimek, Wiesbaden. Bis spätestens 1. Dezember 2010 müssen die Firmen für Produkte, für die eine Zulassung angestrebt wird, ein Zulassungsdossier einreichen. Daraufhin wird das PEI bis Juli 2011 einen Mängelbericht an die Hersteller senden, die diese Mängel - mutmaßlich vor allem fehlende klinische Studien, so Klimek - beseitigen müssen. Die Firma Novartis hat entschieden, dass Depigoid ${ }^{\circledR}$ - und Depiquick $^{\circledR}$-Produkte das Verfahren durchlaufen werden. Bis zur Entscheidung über die Zulassung - spätestens 2016 - sind diese Produkte wie andere in diesem Prozess befindlichen damit weiter rezeptierbar. „Auf viele Mischungen werden Sie aber verzichten müssen“, prophezeite Klimek, ,die Entscheidung über den Eintritt in das Zulassungsverfah- ren ist ja in allen Unternehmen bereits gefallen." Nicht betroffene, seltenere Allergene wie Kräuter, Tierepithelien und Schimmelpilze sind weiterhin als Individualallergene rezeptierbar und nicht von der Neuregelung betroffen, soweit sie nicht in $\mathrm{Mi}$ schungen mit den oben genannten Allergenen angeboten werden.

$\mathbf{f k}$

Industriesymposium „Therapie und Abrech nung - Was gibt's Neues bei SCIT?" im Rahmen des 4. Gemeinsamen Deutschen Allergiekongresses, Berlin, 4. September 2009 Veranstalter: Novartis, Nürnberg

\section{Leistungsstarke Kompaktdesinfektoren}

- Zur MEDICA 2009 präsentierte Miele zwei 90 Zentimeter breite Reinigungs- und Desinfektionsautomaten für den Einsatz in Kliniken und Praxen. Die Standgeräte PG 8535 und PG 8536 sind mit technischen Highlights ausgestattet, die bislang ausschließlich den Großraumdesinfektoren vorbehalten waren. So verfügen beide Geräte über eine integrierte Spülarmkontrolleinheit, die während der Aufbereitung die exakte Drehzahl jedes einzelnen Spülarms überwacht und dokumentiert. Damit lassen sich Beladungsfehler unverzüglich erkennen und beheben, die andernfalls zu Spülarmblockaden und damit zu einer Beeinträchtigung des Reinigungsergebnisses führen könnten.

Die Umwälzleistung beträgt 400 Liter pro Minute beim PG 8535 sowie beachtliche 600 Liter beim PG 8536. Den hygienischen Abschluss aller Aufbereitungsprogramme bildet die Hochleistungstrocknung „Perfect HEPA Drying“.

Mit seiner Höhe von 82 Zentimetern ist der unterbaufähige PG 8535 beispielsweise auch in kleineren Arztpraxen problemlos unterzubringen. Dennoch bietet sein Spülraum Platz für vier DIN-Siebe, zwei AN-Sets, ein bis zwei MIC-Sets oder 48 Gyn-Spekula. Eine deutlich größere Kapazität hält der 117,5 Zentimeter hohe PG 8536 bereit, der gleichzeitig sieben DIN-Siebe oder drei ANSets aufbereiten kann. Auf Wunsch sorgt das absolut wartungsfreie Leitfähigkeitsmesssystem „Perfect Pure Sensor“ dafür, dass Rückstände und unerwünschte Inhaltsstoffe in der Spüllauge schnell erkannt und durch zusätzliche Programmschritte beseitigt werden. Verkaufsstart für beide Geräte ist Anfang 2010.

Nach Informationen von Miele, Gütersloh

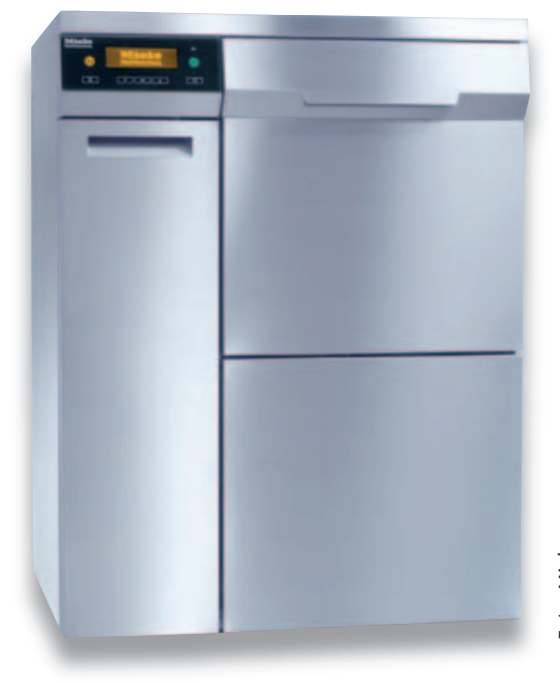

Der PG 8536 von Miele

\section{Compliance bei SIT stärken}

— Die spezifische Immuntherapie (SIT) ist die einzige kausale Therapie allergischer Erkrankungen. Laut den Leitlinien sollte die SIT für den maximalen Therapieerfolg an mindestens drei aufeinanderfolgenden Jahren durchgeführt werden. Jedoch ist die Therapietreue oft unzureichend. Nur ein Viertel bis ein Drittel der Patienten führen eine SIT auch im dritten Jahr noch durch. Um die Therapietreue der Patienten zu erhöhen könnte sich der THErapie-Organizer für Allergiker (THEO) als wichtiger Helfer erweisen. Dieser hochwertige Allergietherapie-Pass wurde speziell entwickelt, um den Patienten zur Durchführung einer dreijährigen Hyposensibilisierung zu motivieren. In diesem Serviceartikel wird nicht nur im Organizer-Teil detailgenau die gesamte Behandlung dokumentiert, sondern er bietet darüber hinaus noch viele Informationen. Der Allergie-THEO passt bequem in jede Tasche und trägt durch persönliche Ansprache seinen Teil zur Motivation der Patienten zum Durchhalten einer vollständigen SIT bei. THEO kann kostenlos angefordert werden unter Tel. 040 727650, Fax 0407227713 , oder E-Mail: info@allergopharma.de.

Nach Informationen von

Allergopharma, Reinbek 